\title{
EFFECTS OF SOURCE ASYMMETRY ON RADIATION \\ TRANSPORT IN INFINITE AIR*
}

B. J. McGregor**

Oak Ridge National Laboratory

Oak Ridge, Tennessee

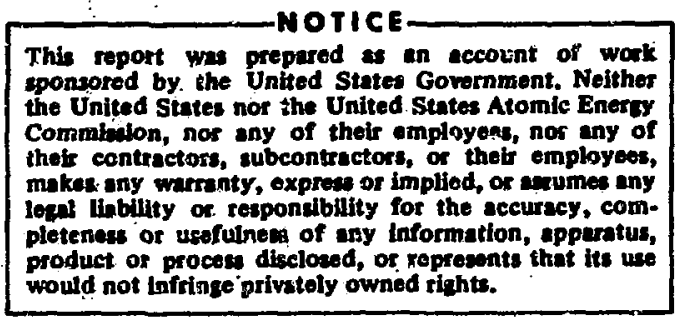

* Research sponsored by the U.S. Atomic Energy Commission under contract with the Union Carbide Corporation.

**Attached to Neutron Physics Division, ORNL from the Australiam Atomic Energy Commission. 
The Defense Nuclear Agency program is concerned with predicting the time integrated and peak radiation dose rates from real devices which are highly asymmetrical in radiation leakage. The time-integrated responses measure the total kerma or tissue dose per source neutron as a function of position. The time-dependent responses, and in particular the peak rates, are important as the peak rate affects the damage to electronics systems. In previous studies both time distributions and integrated responses have been obtained for symmetric point sources in infinite air.

The effects of spatial source asymmetry are easily understood qualitatively. If the source is peaked in a particular direction the fluence or dose will be higher in that direction and the fraction of the fluence or dose due to uncollided source neutrons will also be higher. As the distance from the source increases the effects of source asymmeiry are expected to disappear and the distributions will approach those from a symmetric source.

The time distributions of the neutron responses will have distinct peaks where the response is mostly due to uncollided source neutrons. The time distributions of the gamma-ray responses would be expected to be less affected by the neutron source asymmetry since the secondary gamma-ray production is isotropic about the point of the neutron interaction.

This study, conducted by E. A. Straker and myself, had two main aims. The first was to examine a few extremely asymmetric sources and quantitatively evaluate the effects outlined above. The second ajin was to develop methods which can be used to calculate any asymmetric source distribution.

The three source spatial distributions chosen are called symmetrical, beam, and pancake as shown in Fig. 1. The beam source is nearly the most asymmetrical source possible with all the source neutrons being emitted in a narrow beam about a single direction. The pancake source is less asymmetric as source neutrons are emitted in a small angular spread about a source plane. Many asymmetric distributions could be represented as linear combinations of the three chosen distributions 
time-integrated neutxon ailr kelma at 300 meters for one source neutron (the area under all time gxaphs is unity). The peak rate is cbtained by multiplying the value from the graph by the normalization factor. The threshold is due to the fact that neutrons cannot reach the detector at 300 meters before a certiain time. The peak is due to source neutrons born in the direction of the detector which arrive at the detector without suffering a collision. Figure 3 shows the timedependent neutron air kerma at 300 meters for the beam source. The solid line is the spectra in the source direction while the dotted line is for a direction normal to the source direction. As expected the spectra in the source direction is more peaked and that in the normal direction less peaked than that from a symmetrical distribution. However, the shapes are not dramatically different (the peak rates vary by a factor of 3 ) and the main effert of the source asymmetry appears in the normalization factors which are again the time-integrated responses. These vary by a factor of about 40 giving a combined factor in the peak rate of 120 between thr kerma. In the source and normal direction. Figure 4 shows the time-de ndent air kerma for the paricake source, in the source direction and nearly normal to it. The shapes of the two distributions are very similar to these from the beam source; the main difference in the peak rates comes from the normalization which gives a factor of 10 , giving a difference in peak rates of about 30 . Figure 5 shows the time-dependent gamma-ray air kerma for the symmetric source: Because of the greater speed of ganma rays the threshold is below 1 usec. The distribution is almost horizoncal from 1 to 8 lisec and then falls more steeply than any of the neutron distributions. Figure 6 shows the gamma-ray results for the beam source. Again the solid line is in the source direction and the dotted line is in a direction normal to the source direction. The peaks on the graphs are approximately equal but the normalizations give a factor of 7 in the peak rates. To summarize the time distributions, the peak rates in different directions are affected mostly by the changes in the total response with a lesser effect due to changes in shape.

As in most large-scale calculations it is desirable to calculate the problem by two independent methods as a check on accuracy. This 


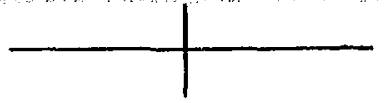

was not entirely possible in this case as the time distributions are only obtainable with a Monte Carlo code but we planned to compare the time-integrated MORSE results with two-dimensional calculations made with the DOT-UNCL codes.

As the study proceeded it was realized that the comparison calculations could be made simpler by using the properties of the adjoint function. Since a dose detector response is isotropic, an adjoint onedimensional ANISN calculation was performed using an isotropic source at the detector point which had an energy dependence according to the detector response function. The adjoint calculation produces an importance function for each neutron or gamma-ray energy group as a function of distance from the source. At eacb point in space the importance function is given for each polar angle increment with respect to the source detector axis. In the ANISN formulation the radius vector corresponds to the direction between the source and the detector.

Once the adjoint solution is obtained for a given response function, any asymmetric source can be calculated by convoluting the angular importance function with the appropriate source.

$$
R(r, \theta)=\sum_{g} \int S\left(\Omega^{1}\right) \phi_{g}\left(r ; \Omega^{1} \cdot S \ell \cdot d \Omega^{1}\right.
$$

$R$ is the response at distance $r$ and angle $\theta$ to the $z$ axis; $S\left(\Omega^{2}\right)$ is the fraction of the source in the solid angle d $\Omega^{3}$ about $\Omega^{l} ; \Omega^{0}$ is the airection between the origin and the detector position $(r, \theta) ; \Omega^{1} \cdot \Omega^{0}$ is the cosine of the angle between $\Omega^{1}$ and $\Omega^{0} ; \phi_{g}(r, \mu)$ is the importance function at radius $I$ and cosine $\mu$ in the energy group $g$.

A program was written to evaluate this equation for the three source distributions and produce isoresponse curves as a function of height above the source and horizontal distance from the source. The timeIntegrated MORSE results were also produced on these graphs.

Figure 7 shows isokerma curves for neutron alr kerma from the symmetric source. The solid line is the integration of the ANISN adjoint 


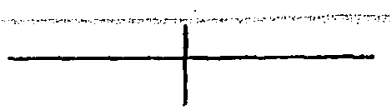

results while the dotted line is results from MORSE. The small irregularities in the dotted results are due to statistics. The agreement between the two methods of calculation is very good. Figure 8 shows the neutron air kerma results for the beam source. The irregularities in the MORSE results are due to the finite angular bin sizes. The point values are from the DOT calculations. The agreement between the MORSE, ANISN, and DOT forward direction results is again good. The DOT calculations at backward angles do not agree very well with the other two methods. It is thought that a finer mesh spacing may remove this discrepancy but further investigation is required. As an index of the effects of source asymmetry the air kerma at 500 meters directly above the source is approximately 50 times the value at 500 meters directly below the source. Figure 9 shows the neutron air kerma for the pancake source. All three methods (MORSE, ANISN, and DOTO agree quite well. The asymmetry factor (ratio of kerma at 500 meters in source direction to that at 500 meters thermal to it) is about 6 .

Figure 10 shows the gamma-ray results for the symmetric source. The curves are further apart in the gamma-ray case illustrating the larger mean free path of gamma rays as compared to the neutron mean free path. At about 500 meters from the source the air kerma from neutrons and secondary gamma rays are approximately equal; beyond this distance, the gamma-ray kerma dominates. This contrasts with the tissue dose where the neutron dose is larger than the gamma-ray dose out to about 1800 meters from the source. Figure 11 shows gamme-ray results from the beam source. As expected, the gamma-ray air kerma shows less effects due to source asymmetry than the corresponding neutron air kerma. For the beam source the asymmetry factor at 500 meters is 13 .

Apart from quantitatively detarmining the effects of source asymmetry in two cases, this study also illustrated the edvantages of using the convoluted ANISN adjoint method when the detector response is isotropic. The use of the properties of the adjoint essentially results in replacing a number of two-dimensional problems for each angular distribution with one one-dimensional calculation. 


\section{References}

1. B. J. McGregor and E. A. Straker, "A Study of the Effect of Source Asymmetry in Time-Dependent Neutron and Secondary Gamma-Ray Transport in Air," to be published as report number ORNL-4741.

2. E. A. Straker, P. N. Stevens, D. C. Irving, and V. R. Cain, "The MORSE Code - A Multigroup Neutron and Gamma-Ray Monte Car1o Transport Code," ORNL-4585 (1970).

3. E. A. Straker and M. L. Gritzner, "Neutrun and Secondary GammaRay Transport in Infinite Homogeneous Air," ORNL-4464 (1969). 


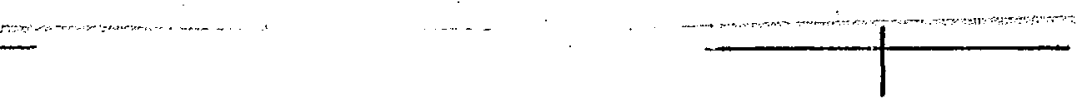

Figure Captions

Fig. 1. Source Angular Distributions.

Fig. 2. Time-Dependent Neutron Air Kerma at a Radius of 300 Meters for a 12.2- to 15-MeV Symmetric Source in Air.

Fig. 3. Time-Dependent Neutron Air Kerma at a Radius of 300 Meters in the Source Direction and at $90^{\circ}$ to the Source Direction for a 12.2to 15-MeV Beam Source.

Fig. 4. Time-Dependent Neutron Air Kerma at a Radius of 300 Meters in the Srource Plane and at $20^{\circ}$ from the $z$ Axis for a 12.2- to 15-MeV Pancake Source in Air.

Fig. 5. Time-Dependent Gamma-Ray Air Kerma at a Radius of 300 Meters for a 12.2- to 15-MeV Symmetric Source in Air.

Fig. 6. Time-Dependient Gamma-Ray Air Kerma at a Radius of 300 Meters In the Sourca Nirections and at $90^{\circ}$ to the Source Direction for a 12.2- to 15-MeV Beam Source.

Fig. 7. Neutron Air Isokerma Curves for a 12.2- to 15-MeV Symmetric Source in Air.

Fig. 8. Neutron Air Isokerma Curves for a 12.2- to 15-MeV Beam Source in Alr.

Fig. 9. Neutron Air Isokerma Curves for a 12.2- to 15-MeV Pancake Source in Air.

Fig. 10. Gamma-Ray Air Isokerma Curves for a 12.2- to 15-MeV Symmetric Source in Air.

Fig. 11. Gamma-Ray Alr Isokerma Curves for a 12.2- to 15-MeV Beam Source in Air. 


\section{ORNL-DWG 71-11067}

SYMMETRIC SOURCE

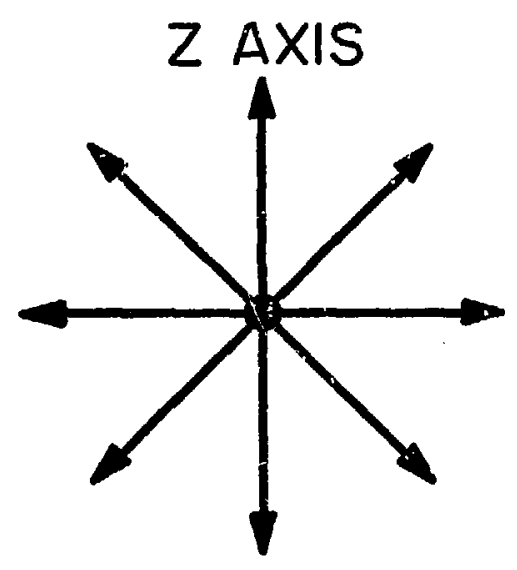

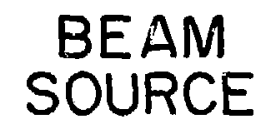

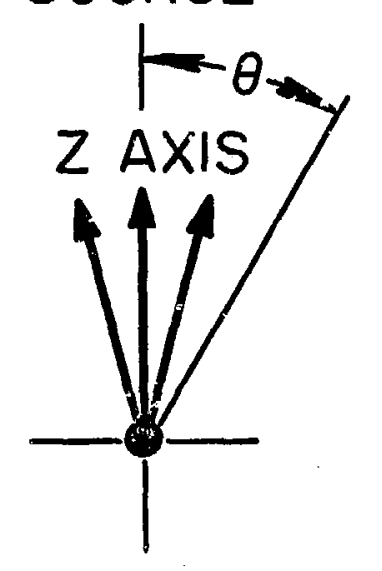

PANCAKE SOURCE

Z AXIS

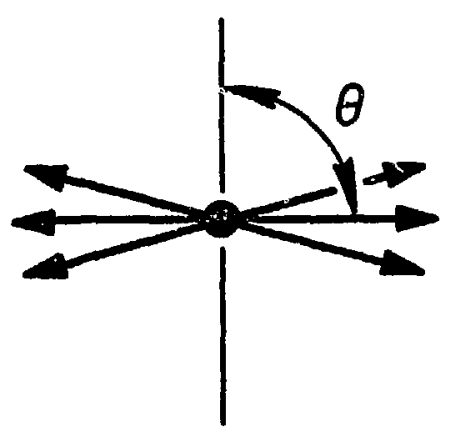

Fig. 1. Source Angular Distributions. 


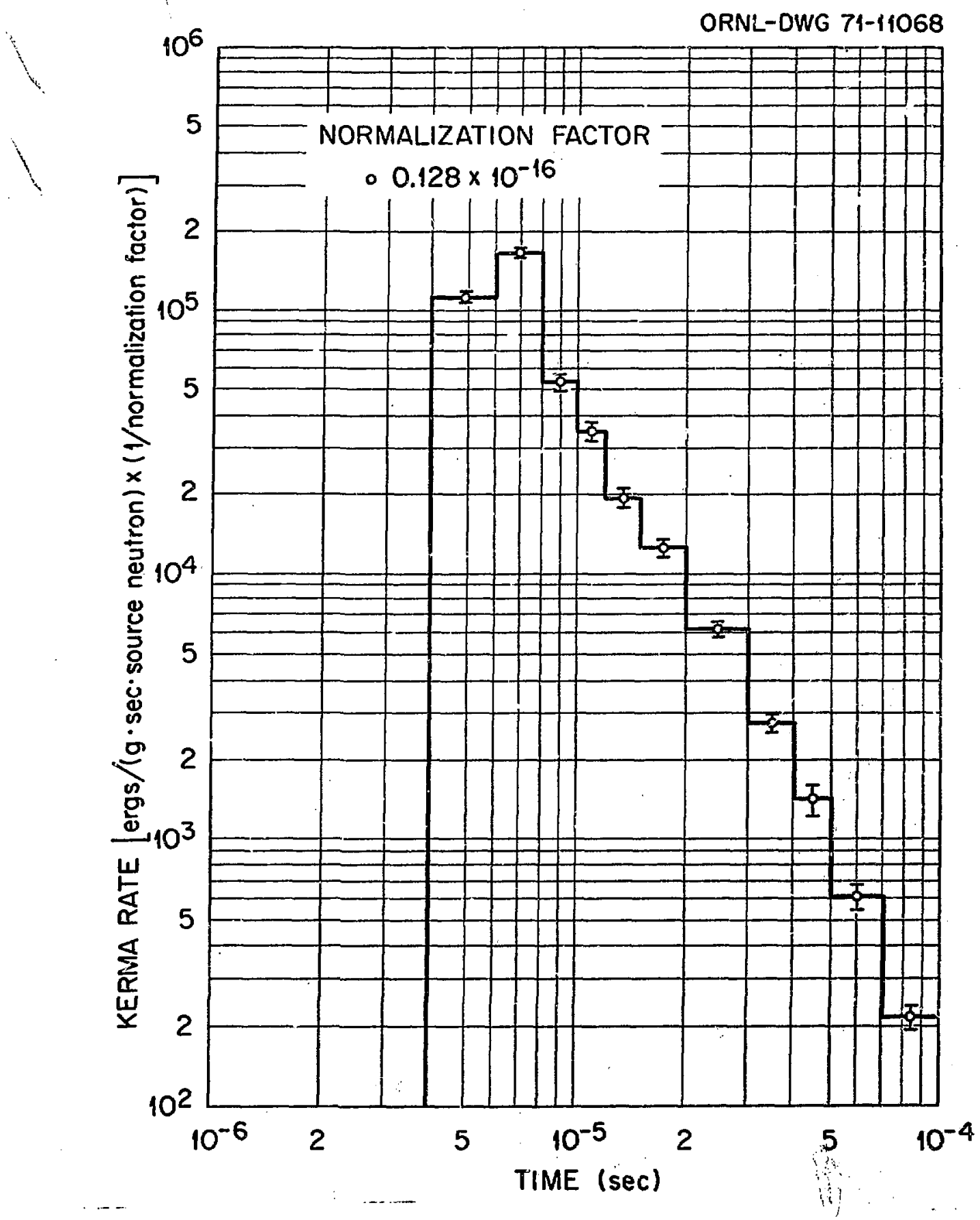

Fig. 2. Time-Dependent Neutron Air Kerma at a Radius of 300 Meters for a 12.2- to 15-MeV Symmetric Source in Air. 


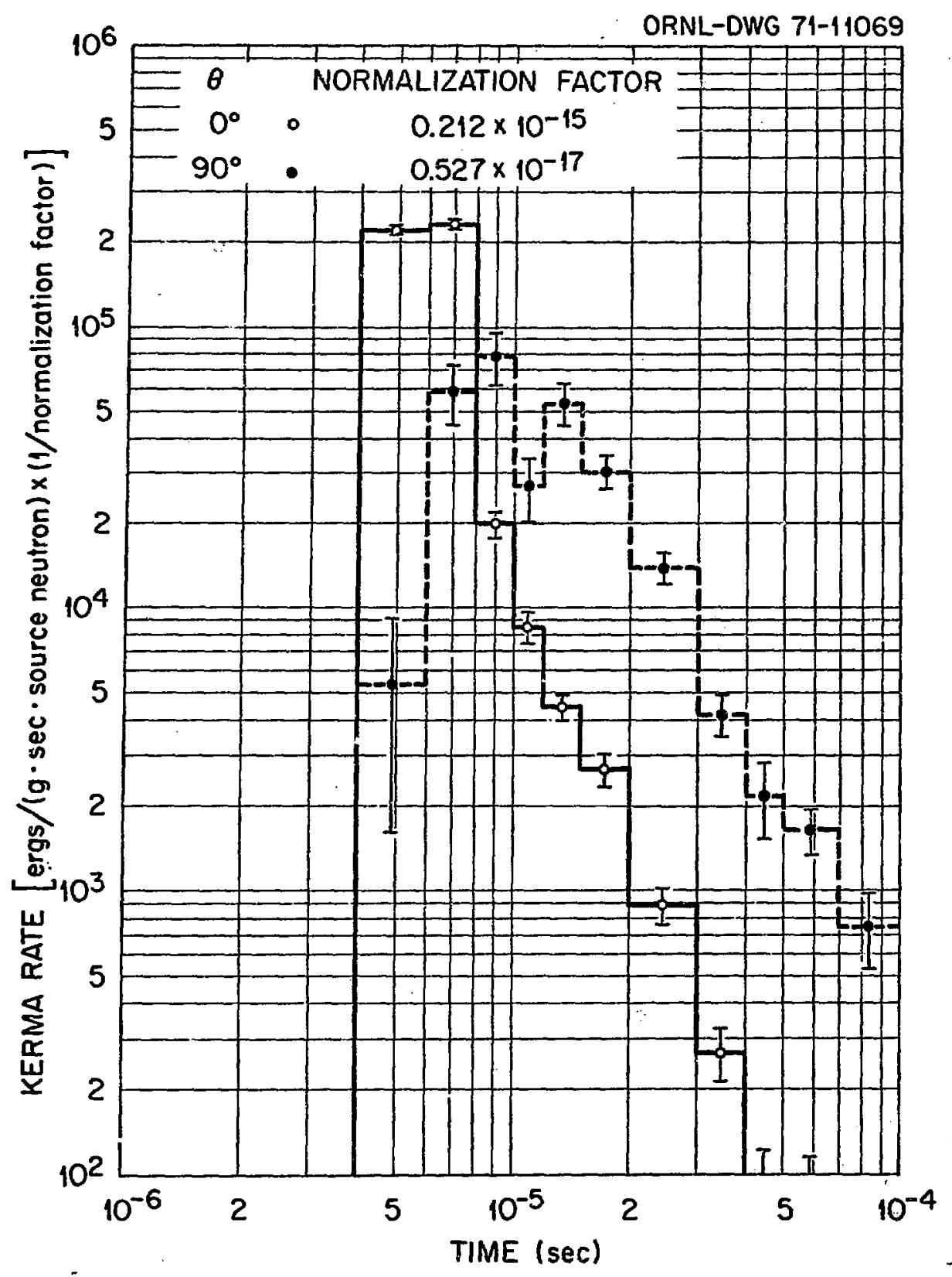

Fig. 3. Time-Dependent Neutron Air Kerma at a Radius of 300 Meters In the Source Direction and at $90^{\circ}$ to the Source Direction for a 12.2to 15-MeV Beam Source. 


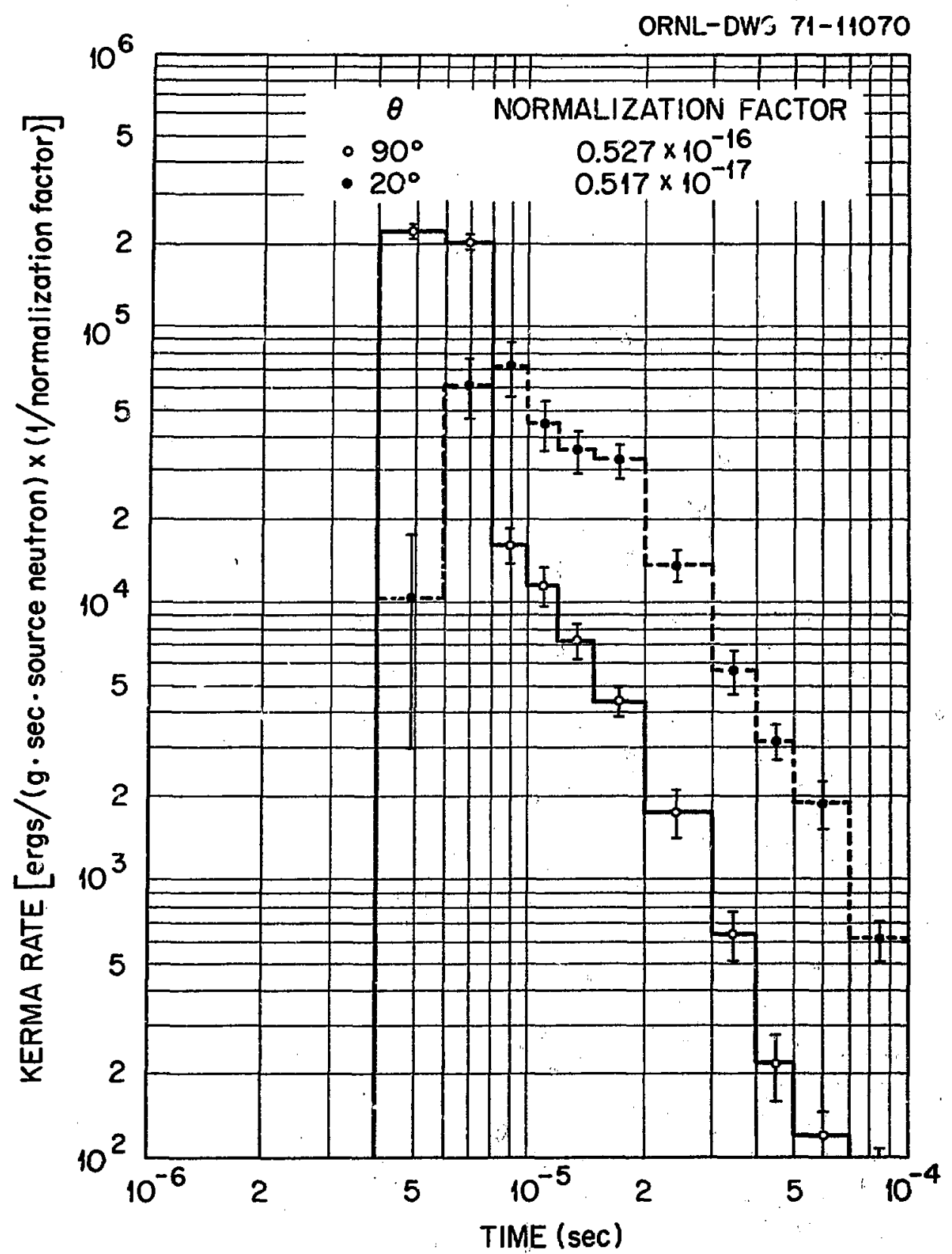

Fig. 4. Time-Dependent Neutron Air Kerma at: a Radius of 300 Meters in the Source Plane and at $20^{\circ}$ from the $z$ Axis for a 12.2- to $15-\mathrm{HeV}$ Pancake Source in Air. 


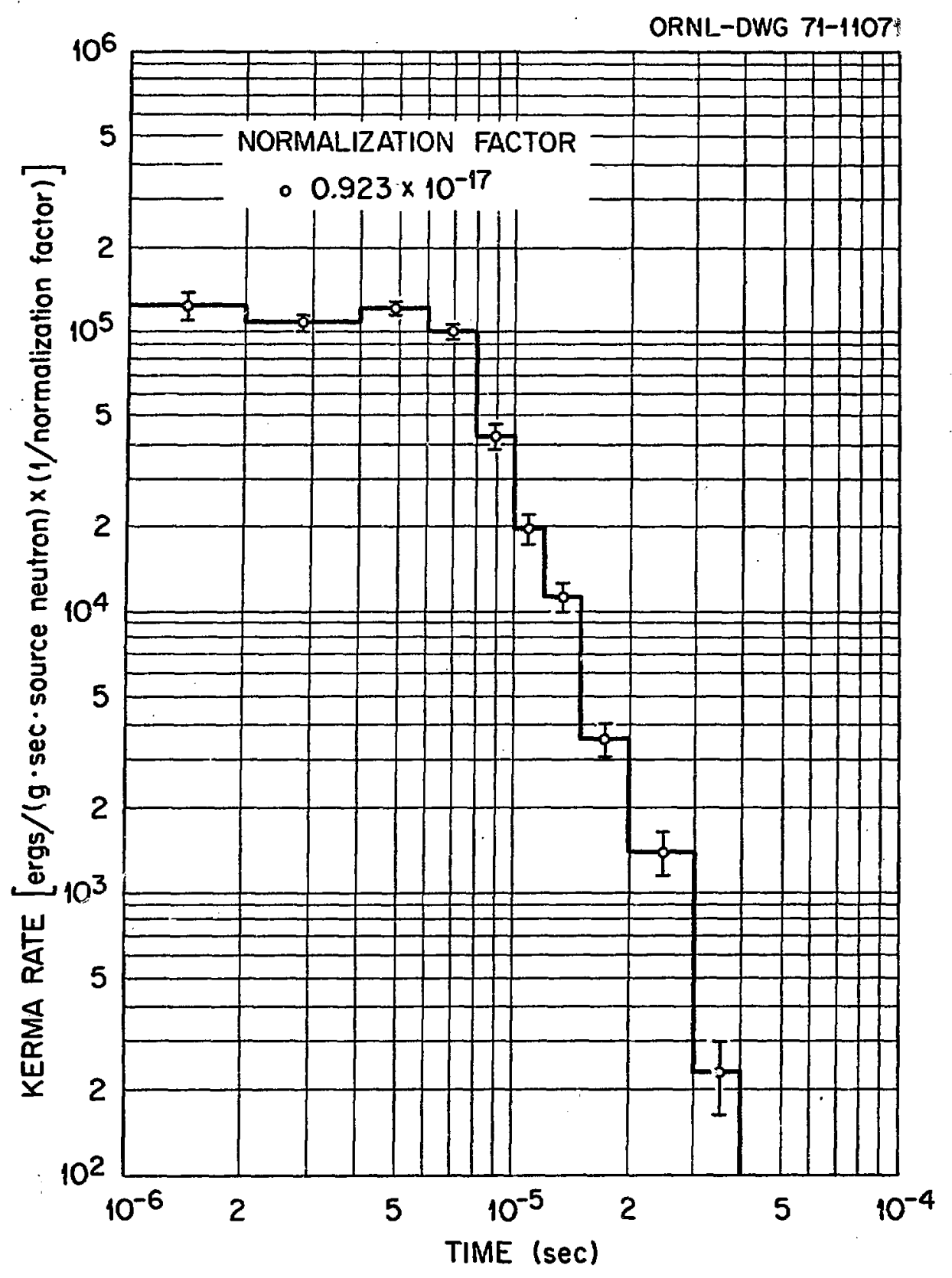

F1g. 5. Time-Dependent Gamma-Ray Air Kerma at a Radius of 300 Meters for a 12.2- to 15-MeV Symmetric Source in Air. 


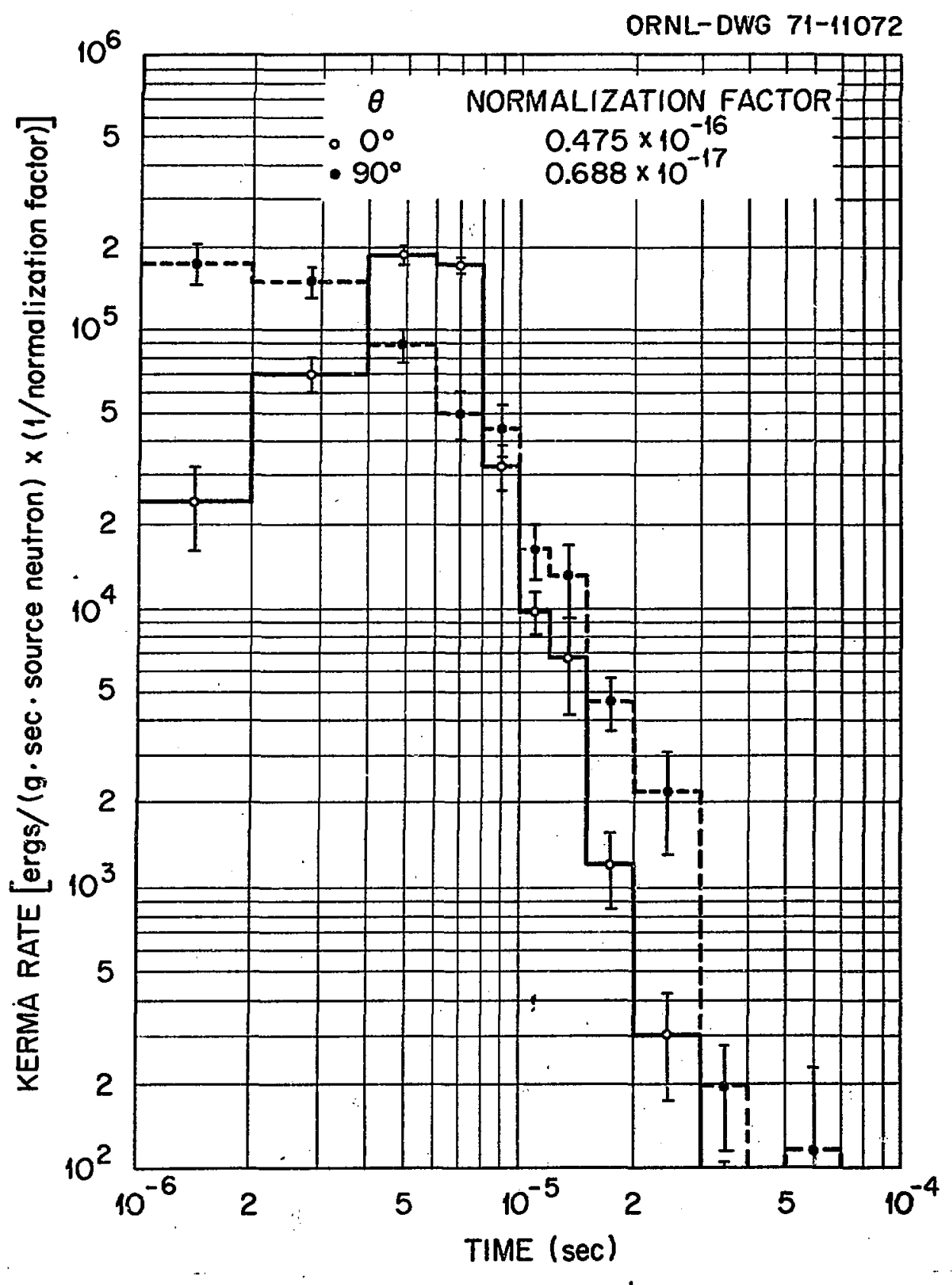

Fig. 6. Time-Dependent Gamma-Ray Air Kerma at a Radius of 300 Meters in the Source Directions and at $90^{\circ}$ to the Source Direction for a 12.2- to 15- MeV Beam Source. 


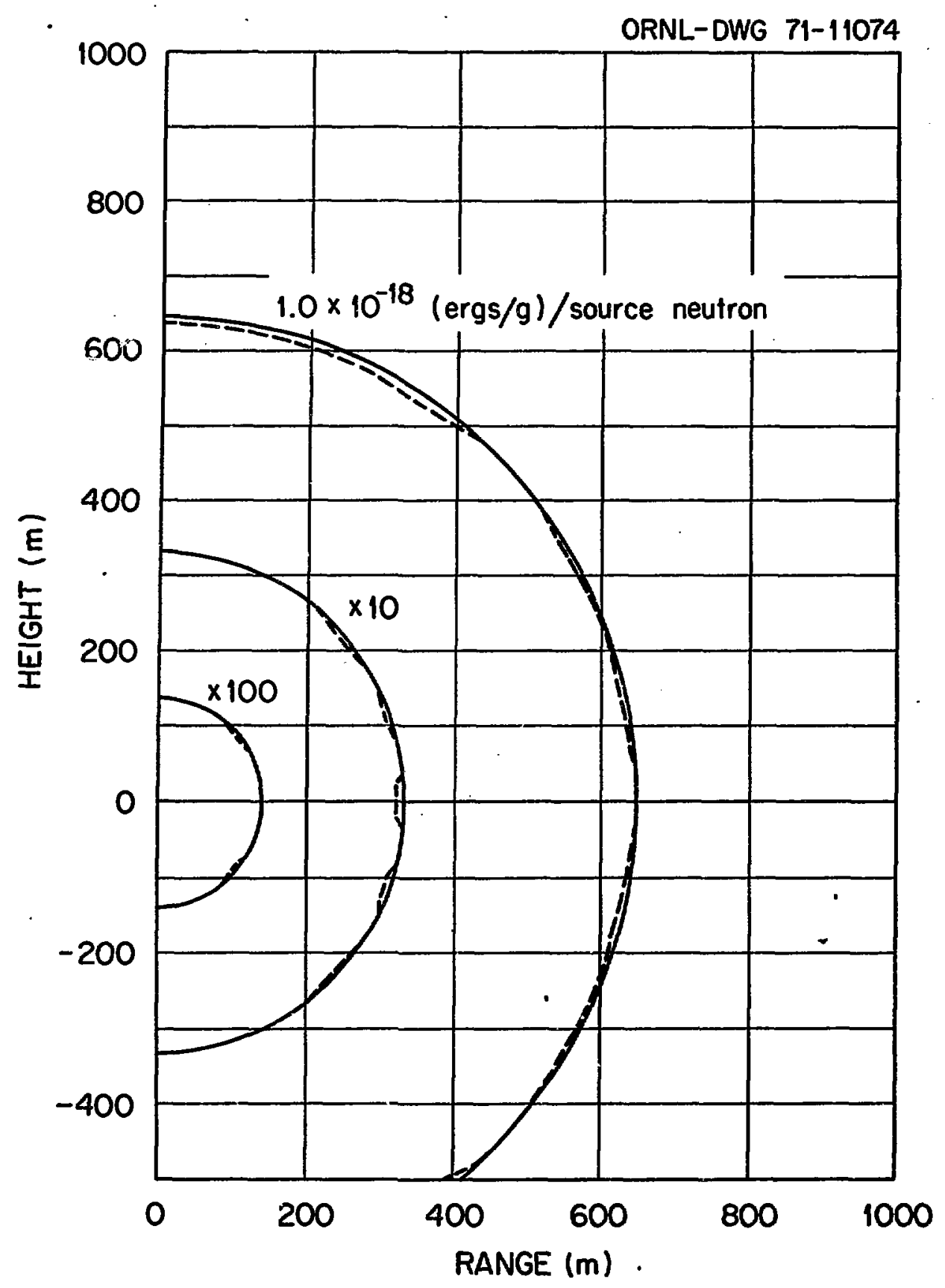

Fig. 7. Neutron Air Isokerma Curves for a 12.2- to 15-MeV Symetric Source :in Air. 


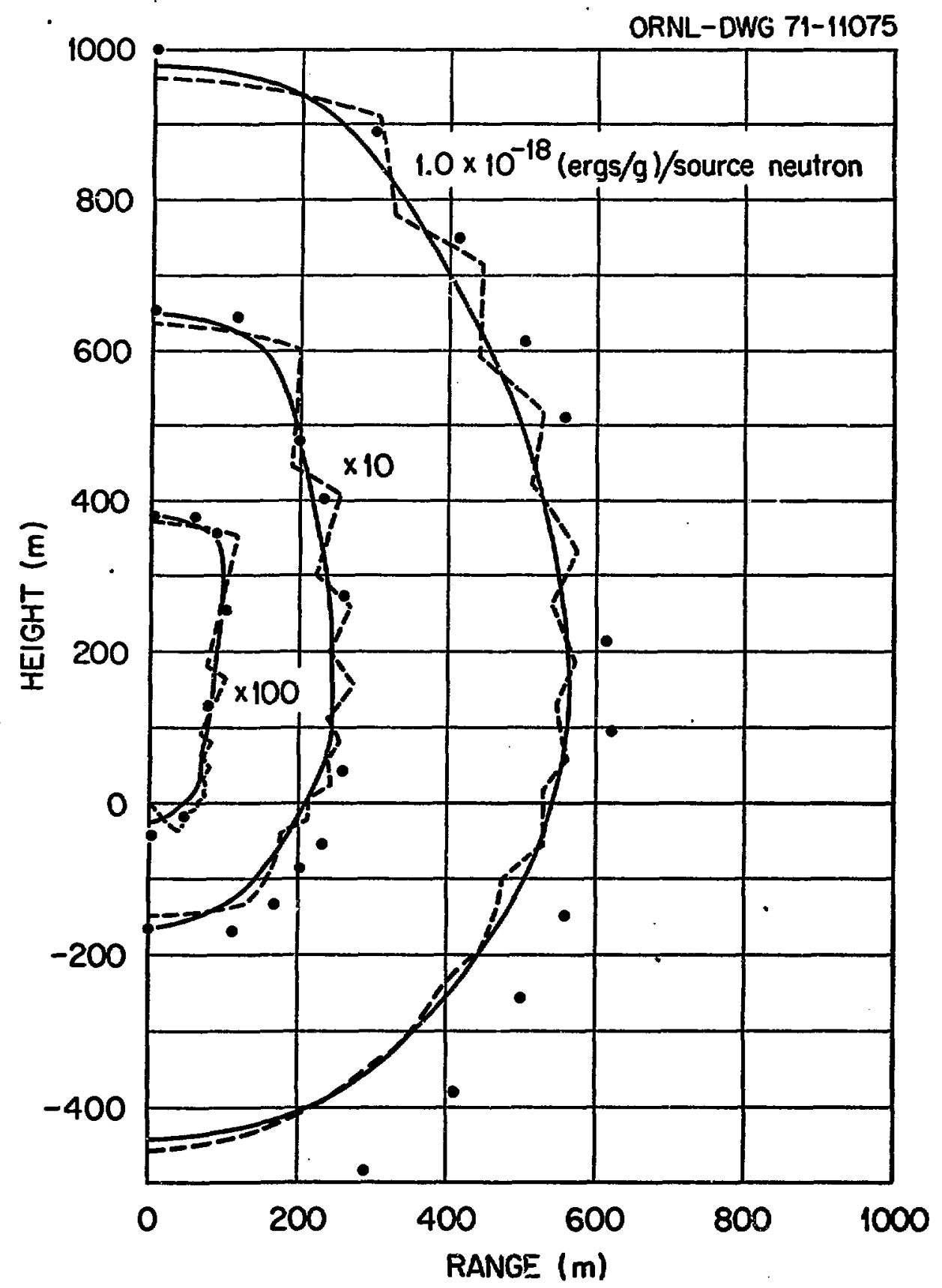

Fig. 8. Neutron Air Isokerma Curves for a 12.2- to 15-MeV Bean Source in Air. 


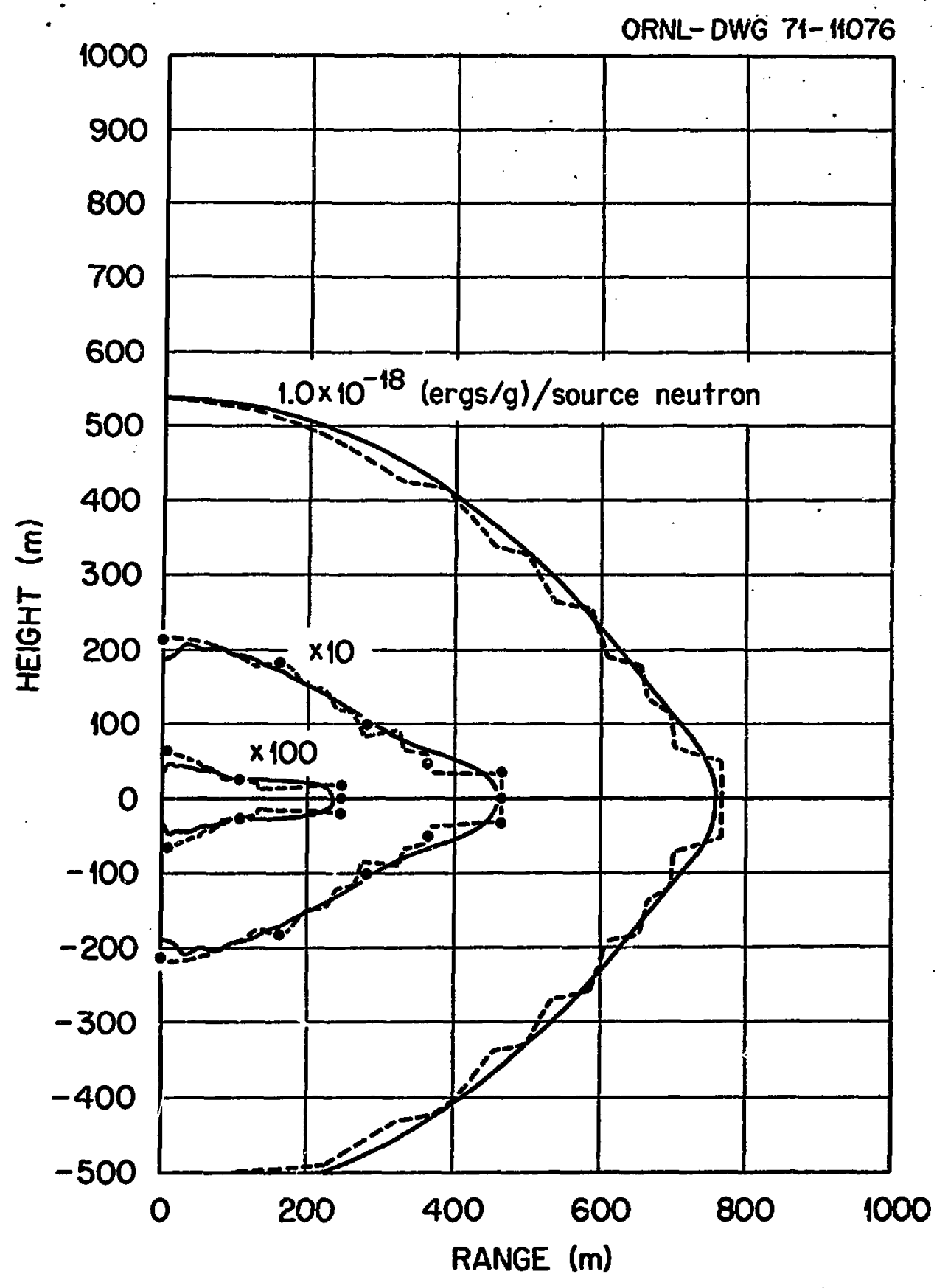

F1g. 9. Neutron Air Isokerma Curves for a 12.2- to 15-MeV Pancake Source in Air. 


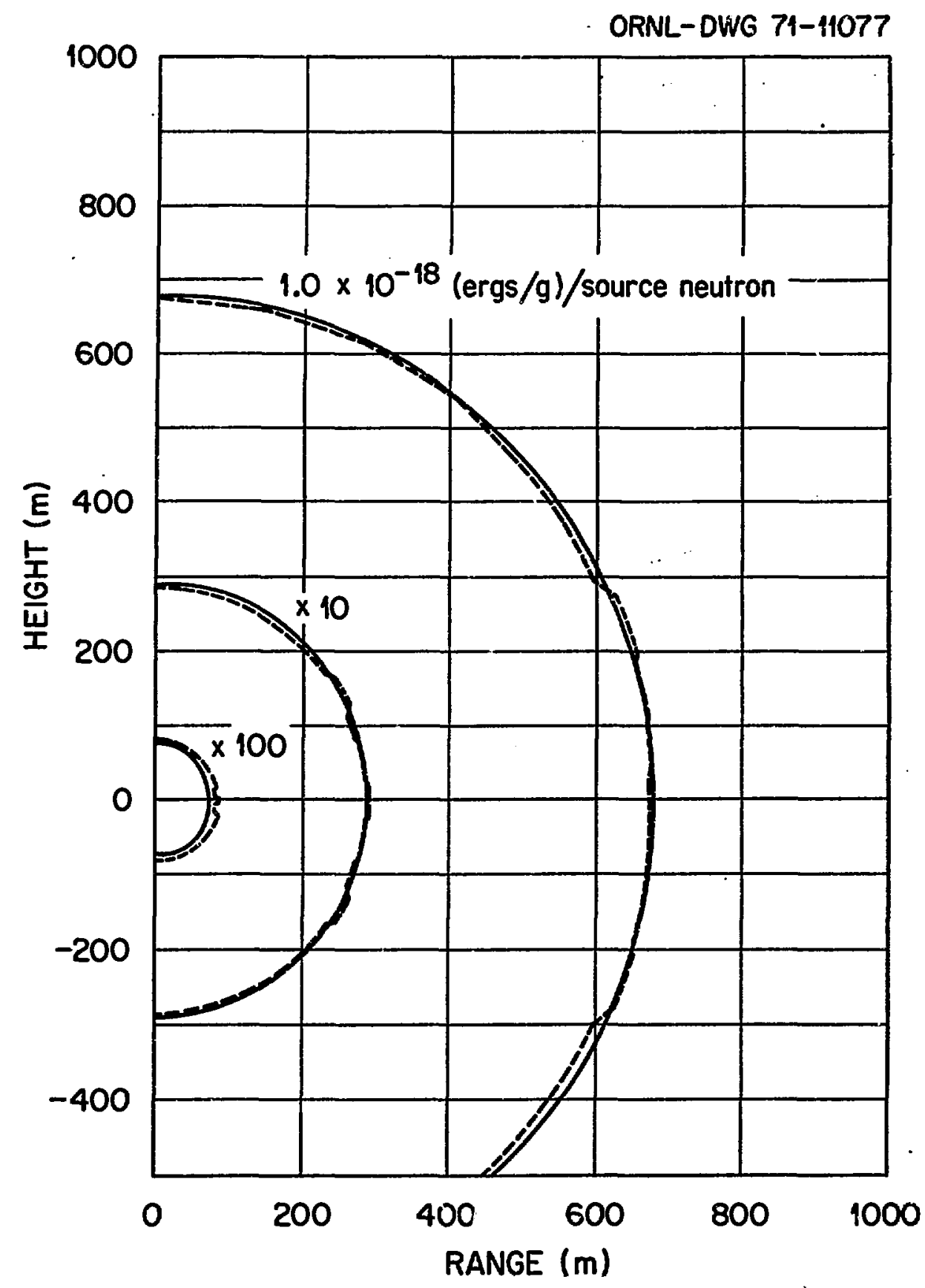

Fig. 10. Gasma-Ray Air Isokerma Curves for a 12.2- to 15- $\mathrm{MeV}$ Symetric Source in Air. 


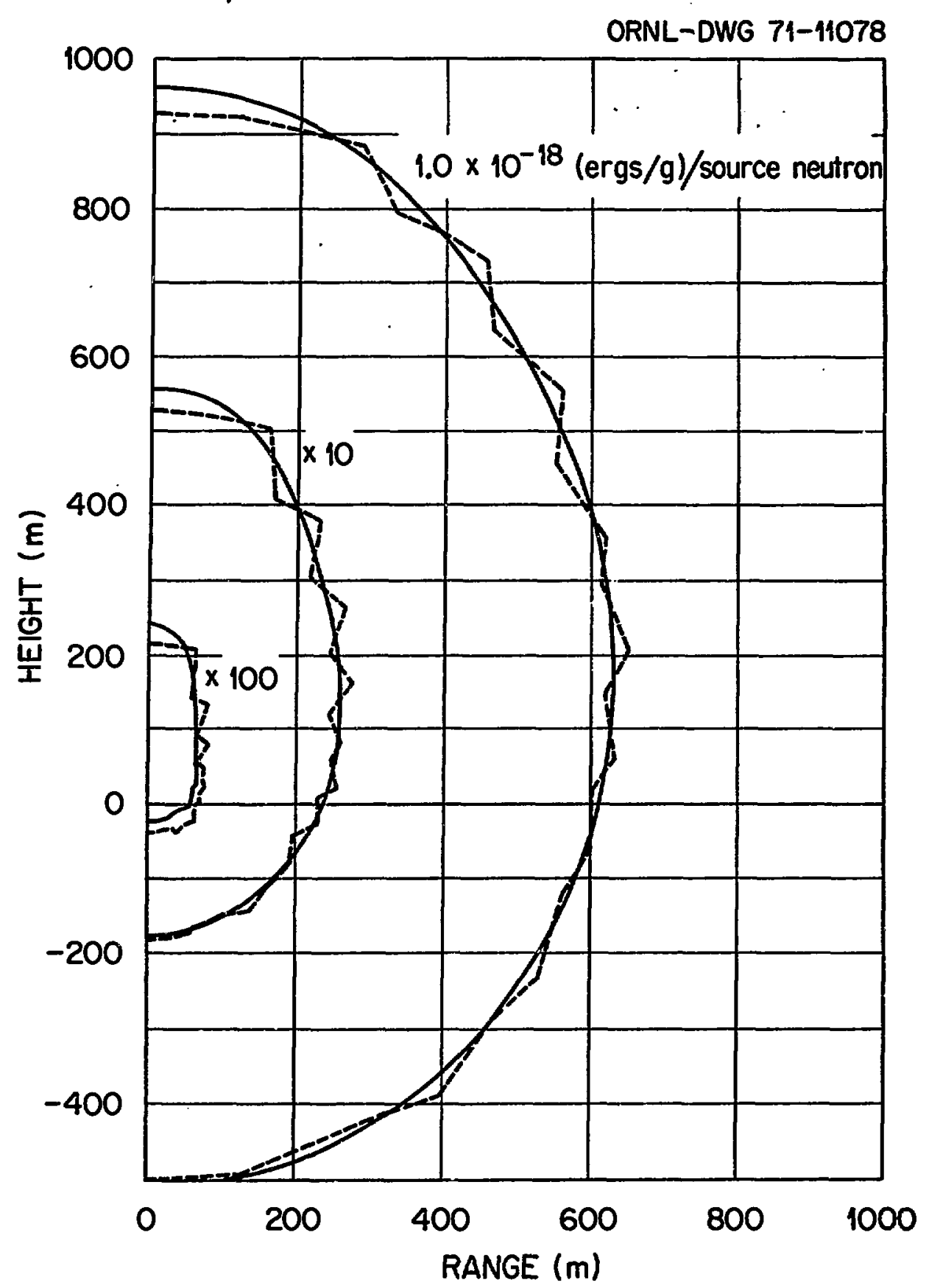

Fig. 11. Gamma-Ray Air Isokerma Curves for a 12.2- to 15-MeV Beam Source in Air. 